\title{
Gold nanoparticles embedded in organic/inorganic hybrid matrix: electrical and electrochemical behavior (Withdrawal Notice)
}

Sandra D. F. C. Moreira, J. P. B. Silva, Carlos J. R. Silva, I. Capan, M. J. M. Gomes, et al.

Sandra D. F. C. Moreira, J. P. B. Silva, Carlos J. R. Silva, I. Capan, M. J. M. Gomes, Manuel F. M. Costa, "Gold nanoparticles embedded in organic/ inorganic hybrid matrix: electrical and electrochemical behavior (Withdrawal Notice)," Proc. SPIE 8766, Nanotechnology VI, 87660W (22 May 2013); doi: $10.1117 / 12.2022359$

SPIE. Event: SPIE Microtechnologies, 2013, Grenoble, France 


\section{Gold nanoparticles embedded in organic/inorganic hybrid matrix: electrical and electrochemical behavior (withdrawal notice)}

Proc. SPIE 8766, 87660W (2013); http://dx.doi.org/10.1117/12.2022359

Online Publication Date: 22 May 2013

Retracted from Publication: 6 June 2013

Conference Date: Wednesday 24 April 2013

Conference Location: Grenoble, France

Conference Title: Nanotechnology VI

Conference Chairs: Rainer Adelung

Sandra D. F. C. Moreira, J. P. B. Silva, Carlos J. R. Silva, M. J. M. Gomes, Manuel F. M. Costa

Univ. do Minho (Portugal)

I. Capan

Institut Ruder Boškovic (Croatia)

This paper has been withdrawn at the authors' request. http://dx.doi.org/10.1117/12.2022359. 